\title{
PHMETRIA ESOFÁGICA EM CÃES
}

\author{
Jorge Luiz Costa Castro ${ }^{1}$ \\ Vinicius Gonzalez Peres Albernaz ${ }^{1}$ \\ Flávio Augusto Vieira Freitas ${ }^{1}$ \\ Ubirajara Iobe Tasqueti ${ }^{1}$ \\ Sérgio Santalucia Ramos da Silva ${ }^{2}$ \\ Alceu Gaspar Raiser ${ }^{3}$
}

\begin{abstract}
RESUMO
A avaliação da phmetria esofágica é um método sensível e especifico para detectar a ocorrência de refluxo gastroesofágico, bem como sua frequência, duração e até mesmo determinar a origem. O conteúdo do refluxo gastroesofágico pode ter origem somente gástrica, duodenal ou de ambos, demonstrando características únicas em relação a valores ácido-base e graus de severidade diferentes. $\mathrm{O}$ objetivo deste trabalho foi determinar o valor do $\mathrm{pH}$ esofágico em cães anestesiados, e avaliar a ocorrência de episódios de refluxo gastroesofágico durante as intervenções cirúrgicas. Foram utilizados nove cães machos e fêmeas adultos saudáveis, com peso corporal médio de 15,0 $\pm 5,4 \mathrm{~kg}$. A pHmetria esofágica foi realizada pela inserção de um cateter pela nasofaringe em 9 cães anestesiados com cetamina, midazolan e metadona; propofol e isoflurano, submetidos as intervenções de mastectomia, orquiectomia e ovariosalpingohisterectomia eletiva. Em nove cães que foram realizadas a passagem do cateter, apenas um animal submetido a ovariosalpingohisterectomia apresentou episódio de refluxo gastroesofágico; comprovado por esofagoscopia. A phmetria foi realizada até 180 minutos pós-anestesia, sem alterações significativas da mensuração do $\mathrm{pH}(\mathrm{P}<0,05)$ em todos os tempos. Os animais foram acompanhados até 30 dias de pósoperatório sem sinais clínicos de esofagite. A média de todas as mensurações de $\mathrm{pH}$ foi de $6.229+-0.5245$.
\end{abstract}

Palavras-chave: $\mathrm{pH}$, esôfago, refluxo, gástrico, anestesia, cães.

\section{ESOPHAGEAL PH-METRY IN DOGS}

\begin{abstract}
Esophageal pHmetry evaluation is a sensitive and specific method to detect gastroesophageal reflux, as weel as its frequency, duration, and origin. Gastroesophageal reflux content can be originated from stomach, duodenal or both, showing unique features of acid-base values and different severity degrees. The aim of this study was to stablish esophageal $\mathrm{pH}$ values in anesthetized dogs, and to evaluate the occurrence of gastroesophageal reflux episodes during surgical intervention. Were used 9 female and male healthy dogs, with mean body weight of $15.0 \pm 5.4 \mathrm{~kg}$. Esophageal pHmetry was performed throght the insertion of a catheter on the nasopharinx in 9 dogs anesthetized with ketamine, midazolam, methadone, propofol e isoflurane, subject to mastectomy, orchiectomy and elective ovariosalpingohysterectomy. Of all $9 \mathrm{dogs}$ in which the catheter was applied, only one animal, subject to ovariosalpingohysterectomy, has shown a gastroesophageal reflux episode, proved by esofagoscopy. pHmetry was performed until 180 minutes post-anesthesia, without
\end{abstract}

\footnotetext{
${ }^{1}$ Pontifícia Universidade Católica do Paraná. Contato principal para correspondência.

${ }^{2}$ Universidade do Sul de Santa Catarina.

${ }^{3}$ Universidade Federal de Santa Maria.
} 
significative changes on $\mathrm{pH}$ mesurement $(\mathrm{P}<0.05)$ at all times. The animals had follow ups until 30 days of post-operative with no clinical signs of esophagitis. The mean $\mathrm{pH}$ measurement was $6.222 \pm 0.5245$.

Keywords: pH, esophageal, reflux, gastric, anesthesia, canine.

\section{PH-METRÍA ESOFÁGICA EN PERROS}

\section{RESUMEN}

La evaluación del pHmetría esofágica es un método específico y sensible para detectar la aparición de reflujo gastroesofágico, así como su frecuencia, duración e incluso determinar el origen. El contenido del reflujo gastroesofágico puede tener origen sólo gástrica, duodenal, o ambos, demostrando características únicas con respecto a los valores de acido-base y distintos grados de severidad. El objetivo de este estudio fue determinar el valor del pH del esófago en perros anestesiados y evaluar la ocurrencia de episodios de reflujo gastroesofágico durante las intervenciones quirúrgicas. Fueron utilizados nueve perros adultos saludables, machos y hembras, con medio peso de $15.0 \pm 5,4 \mathrm{~kg}$. El monitoreo del $\mathrm{pH}$ esofágico fué realizado insertando un catéter a través de la nasofaringe en 9 perros anestesiados con ketamina, midazolam y metadona; propofol y isoflurane, sometidos a intervenciones de mastectomía, orquiectomía y ovariosalpingohisterectomia electiva. En los nueve perros que fueron hechos el paso del catéter, sólo 1 animal sometido a ovariosalpingohisterectomia presentó episodio de reflujo gastroesofágico; probado por esofagoscopia. El monitoreo del pH fue realizado en 180 minutos pós-anestesia, sin cambios significativos en la medición del $\mathrm{pH}(\mathrm{P}<0.05)$ en todos los tiempos. Los animales fueron acompañados hasta 30 días después de la quirugía sin signos clínicos de esofagitis. El promedio de todas las mediciones de $\mathrm{pH}$ fue de 6229 +- 0.5245 .

Palabras clave: $\mathrm{pH}$, esófago, reflujo, gástrico, anestesia, perros.

\section{INTRODUÇÃO}

A medição do pH intra-esofágico é considerado um dos métodos mais sensíveis e específicos para a detecção de refluxo gastroesofágico (RGE) em seres humanos (1); ele permite verificar a presença de refluxo ácido (2). Ocorrência de RGE durante a anestesia é um problema relativamente freqüente nos caninos e um fator de risco para complicações, como pneumonia aspirativa, esofagite e estenoses esofágicas. Os principais fatores envolvidos incluem: pacientes da raça Poodle, posição (Trendelenburg ou decúbito lateral direito), cirurgias ortopédicas que utilizam atropina e morfina como pré-anestesicos, e o uso de propofol como agente de indução (3).

Em cães, pHmanometria esofágica pode ser realizada pela introdução de um cateter para o esófago, equipado com um sensor sensível às flutuações de $\mathrm{pH}$, ligado a um dispositivo de gravação, o que permite a monitorização do $\mathrm{pH}$, bem como da frequência e da duração de episódios de refluxo gastroesofágico. Para posicionar o cateter corretamente, é necessário medir externamente a distância entre os dentes incisivos da mandíbula e a borda cranial da décima costela, com o sensor localizado entre 2 e 7,5 cm da junção esofagogastrica. Outra forma de garantir o posicionamento adequado do cateter é pelo exame radiográfico do tórax, com sua extremidade que aparece entre 2 e 7,5 centímetros cranial à borda costal do diafragma (4).

A avaliação radiográfica e esofagoscopia realizada nas gatas foram fundamentais para confirmar a posição correta do cateter da pHmetria esofágica, e deixou-se excluir o erro de 
posicionamento como uma variável que pode influenciar o resultado. Posicionamento do cateter incorreto, como ocorreria com um cateter dobrado no lúmen esofágico ou com a sua ponta distante do ou dentro do EGS ou do estômago, pode levar a falsas interpretações (5).

Em medicina veterinária, pHmetria é usada para avaliar a incidência de episódios de RGE durante a anestesia. Esta técnica permite identificar episódios de refluxo não visualizados como a regurgitação, bem como para determinar alguns agentes anestésicos que predispõem ao refluxo (6-9).

Estudos mostram que o teor de refluxo no esôfago pode ser originado apenas a partir do estômago (refluxo ácido) ou pode ser uma mistura do conteúdo gástrico e duodeno (refluxo LINA ou gástrica duodenal). Refluxo ácido é o mais frequente. No entanto, refluxo não-ácido pode causar a esofagite mais grave, devido à sinergia entre enzimas gástricas e duodenais $(10,11)$. Nos seres humanos, Nehra et al. (10) afirmam que o refluxo gastro-duodenal para ser mais prejudicial para a mucosa esofageal que refluxo ácido, bem como a concentração total de ácidos biliares no conteúdo refluxo dos pacientes com esofagite erosiva e metaplasia da mucosa esofágica ou estenose esofágica estava significativamente maior do que em pacientes com lesões discretas na mucosa esofágica, o que indica, possivelmente, um sinergismo entre o duodeno e enzimas gástricas.

$\mathrm{O}$ valor do $\mathrm{pH}$ esofágico em cães, por $\mathrm{pHmetria} \mathrm{esofágica} \mathrm{obtida,} \mathrm{imediatamente} \mathrm{após} \mathrm{a}$ indução anestésica, varia entre 5,72 $\pm 1,69$ (7) e 6,42 $\pm 0,65$ (6). No entanto, o pH esofágico é conhecido por diminuir gradualmente durante o procedimento anestésico (7). Este estudo tem como objetivo determinar o valor do $\mathrm{pH}$ esofágico em cães anestesiados, e avaliar a ocorrência de episódios de refluxo gastroesofágico durante as intervenções cirúrgicas e até 6 horas de pós cirúrgico.

\section{MATERIAL E MÉTODOS}

Este trabalho foi aprovado pelo Comitê de Ética em Pesquisa com Animais (CEUA) da Universidade Federal de Santa Maria - RS, sob o protocolo 073/2011; e seguiram os princípios éticos do Colégio Brasileiro de Experimentação Animal (COBEA). O experimento envolveu 9 cães sadios, sem história de sinais clínicos de doenças gastrointestinais, tais como disfagia, regurgitação, vômito e diarréia. Os pacientes foram submetidos aos exames préoperatório para serem submetidos a intervenção cirúrgica de mastectomia, orquiectomia e ovariosalpingohisterectomia eletivas. O exame físico, hemograma e avaliação no soro das atividades das enzimas transferase alanina (ALT), aspartato transferase (AST) e fosfatase alcalina (FA), bem como a concentração de creatinina, foram realizadas em todos os pacientes.

Os pacientes que entraram no experimento foram 9 cães saudáveis machos e fêmeas, com peso entre $7,8 \mathrm{~kg}$ e $15,5 \mathrm{~kg}$, que foram admitidos para as cirurgias previamente marcadas no Serviço de Cirurgia.

Todos os animais foram submetidos a jejum de 12 horas, a privação de água de 6 horas, e ao mesmo protocolo anestésico. Por via intramuscular foi aplicado cetamina $\left(7 \mathrm{mg} \mathrm{kg}^{-1}\right)$ e midazolan $\left(0,3 \mathrm{mg} \mathrm{kg}^{-1}\right)$ associados a metadona $\left(0,3 \mathrm{mg} \mathrm{kg}^{-1}\right)$. Após aproximadamente $10 \mathrm{~min}$, a cateterização da veia cefálica foi realizada, com uma infusão de $\mathrm{NaCl}$ a $0,9 \%(10 \mathrm{ml} / \mathrm{kg} / \mathrm{h})$ sendo realizada a indução com propofol, dose efeito e a manutenção com isoflurano vaporizado com oxigênio a $100 \%$. A CAM (concentração alveolar mínima) do isoflurano também foi dose efeito, ajustada de acordo com o plano anestésico. A frequência cardíaca, frequência respiratória, pressão parcial de corrente final de dióxido de carbono, a pressão arterial sistólica e saturação arterial de oxigênio foram monitoradas.

O cateter de pHmetria foi introduzido no esôfago, pela nasofaringe, imediatamente após a indução da anestesia e intubação orotraqueal e foi assegurada na região rostral da maxila, 
sem alterações em sua posição durante o período de avaliação. Ao mesmo tempo, o eletrodo cutâneo utilizada como referência foi fixado no tórax, numa área tricotomizada de aproximadamente $5 \mathrm{~cm}^{2}$.

Para padronizar e garantir o correto posicionamento do cateter, a metodologia de medição do corpo externa descrito por Waterman e Hashim (4) foi adaptada. Um cateter de pHmetria 2,1 milímetros foi introduzido transnasal. A superfície rostral da narina foi utilizada como referência, em vez dos dentes incisivos da mandíbula. A distância entre a superfície rostral da narina e a borda cranial da $10^{\mathrm{a}}$ costela, foi determinada com os animais em decúbito lateral esquerdo e seu pescoço normalmente estendidos. $\mathrm{O}$ correto posicionamento do cateter foi confirmado em todos os animais por avaliação radiográfica do tórax (4).

Foi aplicado um gel de lidocaína a $5 \%$ sobre o cateter, mas não sobre o sensor de $\mathrm{pH}$ e anestesia local na cavidade nasal, com spray de lidocaína a 10\%, seguindo técnica descrita por Favaratto et al. (12).

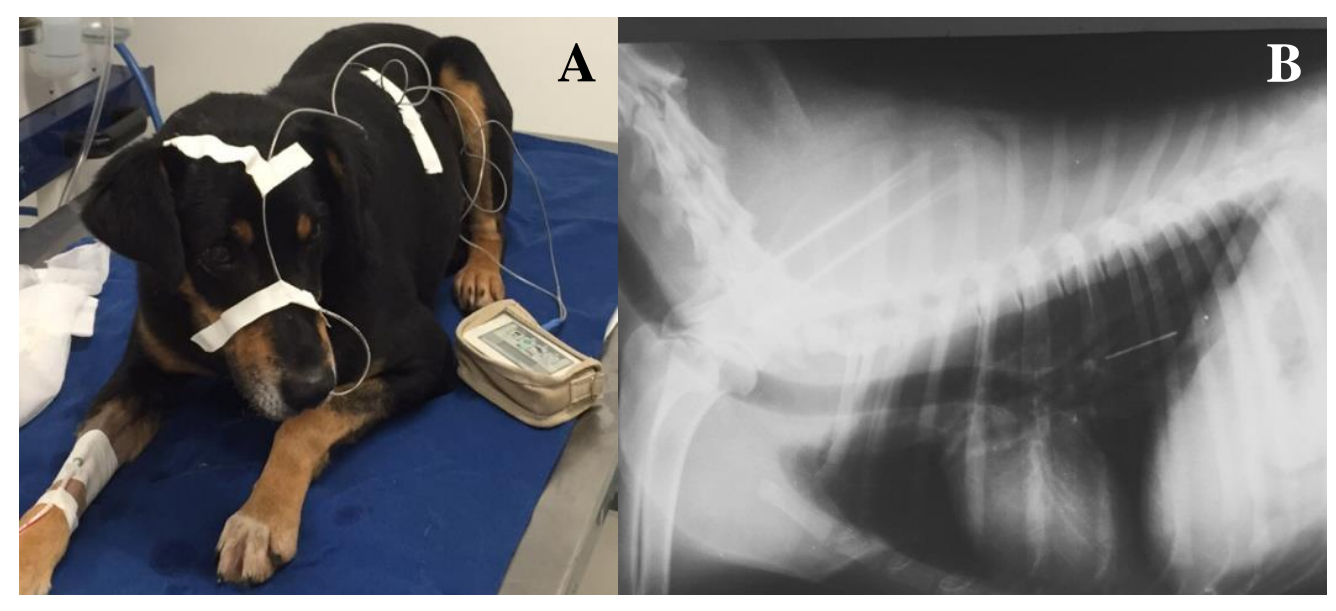

Figura 1. Paciente canino submetido a pHmetria esofagica intraluminal. A) Paciente acordado após 180 minutos da intervenção cirúrgica, no momento da retira do pHmetro esofágico. B) Radiografia lateral de tórax onde visibiliza o catéter intraluminal no esôfago antes do esfincter esofágico inferior (seta branca).

Os pacientes foram mantidos em decúbito dorsal horizontal, sobre uma mesa cirúrgica, durante o procedimento cirúrgico, que durou em média 55 minutos. Após o final da cirurgia, os animais foram mantidos em decúbito lateral, e cuidadosamente observados até à recuperação completa da anestesia.

O aparelho foi calibrado antes de cada experiência utilizando solução tampão (pH 7.0 e 1.0), com a medição do $\mathrm{pH}$ de inicio dentro de 1 hora após a calibração. Os valores de $\mathrm{pH}$ do esôfago intra-operatório foram monitorados e gravados utilizando pHmetria esofágica intraluminal. Assim, um cateter flexível $2.1 \mathrm{~mm}$ (Dynamed ${ }^{\circledR)}$ com um único sensor pH ligado a um dispositivo de gravação foi introduzido no esófago até que o sensor foi posicionado próximo e cranialmente à junção esofagogastrica localizado.

$\mathrm{O}$ monitoramento do $\mathrm{pH}$ esofágico começou aproximadamente 5 minutos após a indução da anestesia. O momento da primeira avaliação foi classificado como zero (controle), sendo o $\mathrm{pH}$ monitorado continuamente e registrado a cada 30 minutos por um período de 180 minutos. Após 3 horas de monitoramento do pH esofágico pós-anestesia, a sonda foi retirada.

Para a mensuração e registro do $\mathrm{pH}$ foi necessária a tricotomia da região lateral do tórax e colocação do anel adesivo dupla-face para uma melhor fixação do eletrodo de referência na pele do paciente, na altura referente à localização do esôfago torácico caudal. O eletrodo de 
referência foi fixado na parede torácica mediante a interposição de gel na ventosa. O cabo do cateter foi acoplado a um gravador (pHmetro) que registrou todo o traçado e as alterações do $\mathrm{pH}$. Ao final do procedimento, o pHmetro esofágico foi fixado ao tórax por meio de bandagem e adaptado, em cada paciente, colar elisabetano. Após 180 minutos, foi retirado o transdutor e realizada a leitura dos dados no aparelho.

$\mathrm{O} \mathrm{pH}$ esofágico foi mensurado durante todo o procedimento anestésico. Considerou-se como episódio de refluxo gastroesofágico ácido, quando o $\mathrm{pH}$ foi inferior a 4, como indicando o refluxo do conteúdo gástrico, ou um aumento acima de 7,5 foi considerado como evidência de refluxo duodeno esofágico (13). Para a confirmação do refluxo gastroesofágico, foi utilizado um videoendoscópio NTSC, diâmetro externo da ponta distal de $9,7 \mathrm{~mm}$, comprimento de trabalho de $1400 \mathrm{~mm}(1,4 \mathrm{~m})$ e o qual foi conduzido em todos os animais do estudo, imediatamente ao término da cirurgia, para avaliar a presença de refluxo visível no lúmen esofágico, e para confirmar o correto posicionamento da sonda de pHmetria. O cateter foi mantido imediatamente após esofagoscopia, até por 3 horas de pós-operatório imediado.

A medicação administrada no trans-operatório aplicada foi apenas o Meloxican $(0,2 \mathrm{mg}$ $\mathrm{kg}^{-1}$ ) por via intramuscular, e repetida na dose de $0,1 \mathrm{mg} \cdot \mathrm{kg}^{-1}$ por via oral 24 horas após até 4 dias no período pós-operatório.

A média e desvio-padrão foram calculados após agrupar os valores de pH do esôfago para cada 30 minutos observados. Análise de variância (ANOVA) com medidas repetidas foi realizada para comparar os valores médios do $\mathrm{pH}$ esofágico durante a anestesia e até 3 horas após. O valor obtido do $\mathrm{pH}$ esofágico durante a anestesia foi comparado com o $\mathrm{pH}$ durante o pós-anestésico até 3 horas.

\section{RESULTADOS}

A média do $\mathrm{pH}$ mensurado em todos os tempos avaliados foi de $6.229 \pm 0.5245$. A média e o desvio-padrão em cada tempo foi de $6.633 \pm 0.3162$ na indução, $6.389 \pm 0.8753$ no transoperatório, $6.344 \pm 0.510230$ minutos posoperatório, $6.322 \pm 0.396260$ minutos, 6.156 \pm 0.394190 minutos, $6.156 \pm 0.3941120$ minutos, $5.789 \pm 0.3551150$ minutos e $6.044 \pm$ 0.4640180 minutos (Tabela 1). Todos os tempos foram considerados estatisticamente semelhantes $(\mathrm{P}>0.05)$, com exceção das mensurações no momento da indução anestésica e 150 minutos após o término do procedimento $(\mathrm{P}<0.05)$ (Figura 3$)$.

Tabela 1. Média, desvio-padrão, valor máximo e médio

\begin{tabular}{cccccccccc}
\hline & Indução & Transop. & $\begin{array}{c}\mathbf{3 0} \\
\text { Min }\end{array}$ & $\begin{array}{c}\mathbf{6 0} \\
\text { min }\end{array}$ & $\begin{array}{c}\mathbf{9 0} \\
\text { min }\end{array}$ & $\begin{array}{c}\mathbf{1 2 0} \\
\text { min }\end{array}$ & $\begin{array}{c}\mathbf{1 5 0} \\
\text { min }\end{array}$ & $\begin{array}{c}\mathbf{1 8 0} \\
\text { min }\end{array}$ & Total \\
\hline Média & 6.633 & 6.389 & 6.344 & 6.322 & 6.156 & 6.156 & 5.789 & 6.044 & 6.044 \\
DP & 0.3162 & 0.8753 & 0.5102 & 0.3962 & 0.3941 & 0.3941 & 0.3551 & 0.4640 & 0.4640 \\
Máx & 7.0 & 7.0 & 7.0 & 6.8 & 7.0 & 7.0 & 6.5 & 6.7 & 7 \\
Mín & 6.4 & 4.2 & 5.4 & 5.6 & 5.8 & 7.0 & 5.4 & 5.5 & 4.2 \\
\hline
\end{tabular}

Legenda: DP - Desvio-padrão; Transop. - Transoperatório; Min - Minutos; Máx - Máximo; Mín - Mínimo.

Regurgitação foi observada em apenas um dos cães durante a anestesia e em nenhum dos pacientes durante a recuperação anestésica e o acompanhamento pós anestésico.

Considerou-se a queda abrupta de $\mathrm{pH}$ esofágico para valores inferiores a 4 como permitiu a identificação do momento exato em que ocorreu o refluxo ácido; pois em um dos cães, o refluxo ácido ocorreu 30 minutos após a indução da anestesia. $\mathrm{O}$ pH aumentou após este período, mantendo-se entre 4 e 5 , até o final da cirurgia. A ocorrência de refluxo, neste caso, foi confirmada por esofagoscópio no fim do procedimento cirúrgico. 
Esofagoscopia permitiu a identificação de RGE em 1 cão $(11,11 \%)$, pela visualização direta das secreções e conteúdo gástrico no lúmen esofágico. Permitiu também observar as condições da mucosa esofágica. Nos pacientes que não sofrem de refluxo, a mucosa da região distal do esófago apresentou-se de coloração clara e rosea (Figura 2).

No exame clínico realizado no sétimo e no décimo quarto dia após a cirurgia e após 30 dias do pós-operatório, o proprietário informou que o paciente que anteriormente havia sido detectada a ocorrencia de RGE, não apresentou manifestações clínicas compatíveis com esofagite ou estenose de esôfago, evidente pela ausencia de vômito, disfagia, sensibilidade dolorosa e diminuição do apetite.

$\mathrm{O}$ cateter de pHmanometria foi introduzido facilmente nos cães sob anestesia geral, e permitiram a obtenção de valores de $\mathrm{pH}$ de partida após indução de anestesia. Durante a recuperação pós-anestésica, foi necessário a anestesia local da cavidade nasal com lidocaína em conjunto com uso de colar elizabetano em 2 cães. O momento de desconforto no pósoperatório esteve presente quando da manipulação do cateter em 2 pacientes, onde foi necessária uma nova fixação e a manipulação do cateter teve como resposta dos pacientes, uma série de espirros e engasgos, com movimentos da cabeça, que se tornaram cada vez menos freqüente após o cateter ser fixado novamente.

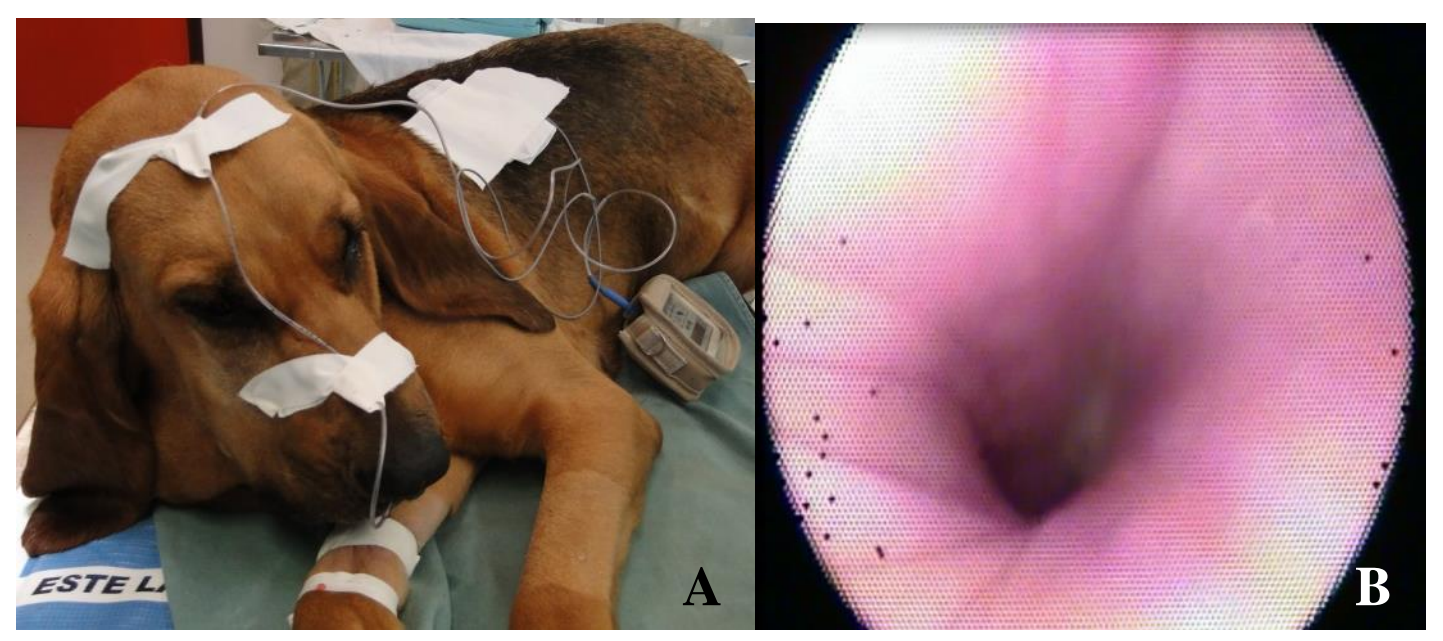

Figura 2. Paciente canino submetido a pHmetria esofagica intraluminal. A) Paciente acordado após 180 minutos da intervenção cirúrgica, no momento da retirada do pHmetro esofágico, note a fixação do cateter ao plano nasal, topo da cabeça e ao flanco torácico. B) Imagem esofágica da esofagoscopia visibilizando o esôfago de coloração rósea clara sem sinais de inflamação ou qualquer alteração patológica.

Sangramento da mucosa nasal de baixa intensidade ocorreu em 4 animais no momento da introdução do cateter. A radiografia de tórax e a esofagoscopia revelou que o cateter foi posicionado corretamente em todos os animais.

Após o período de medição, o cateter foi facilmente removido, ocorrendo espirros em 3 cães no momento da remoção. 


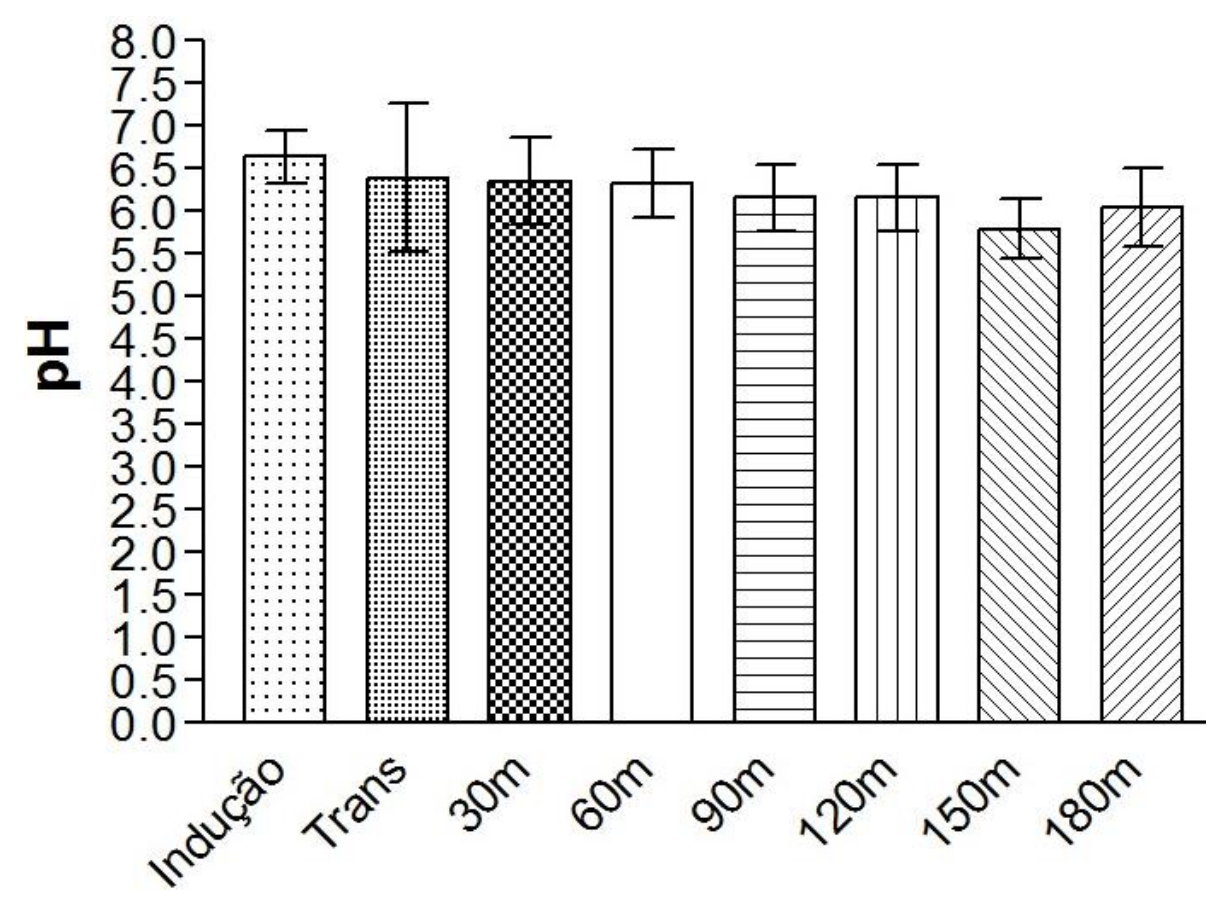

Figura 3. Gráfico com valores da Média e desvio-padrão do valor de $\mathrm{pH}$ em relação ao momento da avaliação. Todos os valores foram considerados sem diferença estatística significativa ( $\mathrm{P}>0.05 \%)$ com exceção de 150 minutos e indução $(\mathrm{P}<0.05 \%)$.

\section{DISCUSSÃO}

Um pH esofágico mais alcalino pode ser evidenciado principalmente em pacientes que estão acordados, devido a deglutição da saliva; o que não acontece em pacientes submetidos a anestesia geral, pois neste caso não há contato do sensor de $\mathrm{pH}$ com a saliva, pois ocorre inibição do reflexo de deglutição pela anestesia, e tem-se como resultado um pH esofágico menos alcalino; bem diferente do que ocorre em cães que estão acordados e isso poderia explicar um pH esofágico superior nos cães acordados após a anestesia (14). Além disso, durante a anestesia, há uma redução do peristaltismo, que associado a uma falta de saliva que neutraliza o $\mathrm{pH}$ ácido, pode contribuir para um desenvolvimento maior de esofagite se ocorrer o RGE (14).

$\mathrm{O}$ valor médio do $\mathrm{pH}$ esofágico em cães anestesiados que não sofreram episódios de refluxo no presente estudo $(6.229 \pm 0.5245)$ foi menor do que o relatado na literatura por Raptopoulos e Galatos (6) $(6,42 \pm 0.65)$; e maior que 5,76 $\pm 0,62$, relatados por Wilson et al. (15), usando sensores de $\mathrm{pH}$ de vidro em vez de sensor de antimônio como utilizado em nosso estudo. Esta diferença nos resultados pode estar relacionada com os diferentes tipos de sensores de $\mathrm{pH}$. Sensores de vidro com referência interna produzem resultados mais confiáveis em termos de tempo de resposta e sensibilidade do que os sensores de antimônio (16). No entanto, ambos são aceitáveis para pHmetria esofágica (1).

No presente estudo, o refluxo gastroesofágico foi detectado em $11,11 \%$ (1/9), resultado semelhante a 13,3\% dos cães anestesiados no estudo de Favaratto et al. (12), incidência estas menores que as observadas em outros estudos com percentuais variando 46-60\% $(9,16,17)$.

É difícil definir qual o tipo de intervenção cirúrgica pode favorecer a um refluxo durante a anestesia. Trabalhos relatam que as cirurgias abdominais podem predispor a uma maior incidência de refluxo por causa de um aumento na pressão intra-abdominal, dentre as quais as cirurgias uterinas é descrita como a causa mais comum de RGE em cães (18). Outros estudos mostraram que a cirurgia gastrointestinal, procedimentos de diagnóstico por imagem, 
ou uma combinação de ambos, durante o mesmo procedimento anestésico podem aumentar o risco de regurgitação. É possível que uma alteração da profundidade da anestesia, e mudanças na posição do decúbito durante o procedimento possa causar RGE (19). No entanto, outro estudo mostrou que RGE ocorreu em $13 \%$ dos cães anestesiados, nenhum dos quais haviam sido submetidos a cirurgia abdominal (20).

A diferença do presente trabalho é que todos estes estudos têm em comum a administração de morfina como parte da medicação pré-anestésica; isto parece razoável quando se leva em consideração a constatação de que a morfina reduz o tônus EGS (21). No presente estudo, não foi utilizada morfina como pré-anestésico e sim cetamina e midazolan. Outro estudo apresentou uma incidência de refluxo em 4,8\% dos animais, bem menor do que essa pesquisa; e os autores relatam o uso de acepromazina, tiopental e halotano (8). Estes autores utilizaram apenas cães fêmeas, e nenhum procedimento cirúrgico foi realizado nos pacientes durante a avaliação, diferente do presente estudo, pois os pacientes foram submetidos a intervenções cirúrgicas.

Episódios de RGE ácido não foram observados em nenhum cão acordado durante o período de observação, o que corrobora a conclusão de que o refluxo ácido é um evento de baixa frequência em cães com junção do esôfago funcional (22). Segundo esses autores, a incidência de refluxo ácido é baixa e ocorre em cerca de $0,98 \%$ (22) no período de observação. No entanto, existe maior probabilidade para os episódios de RGE fisiológicas ocorrer durante ou imediatamente após a alimentação (23), o que não pôde ser avaliado neste estudo, uma vez que os animais não foram alimentados durante o período de observação (até 3 horas pós-anestesia).

No presente estudo, pHmanometria foi realizada ao longo de um período de $4 \mathrm{~h}$, em vez dos habituais $24 \mathrm{~h}$ em seres humanos. Alguns estudos demonstraram que a pHmanometria de 8 horas produz resultados semelhantes ao de pHmetria-24h. Acreditamos que mesmo com o período de observação mais curto (4 horas), o mesmo que recomendado para pacientes que não toleram bem o exame, como as crianças (24-26), os resultados possam ser semelhantes aos obtidos com 24 horas.

A avaliação radiográfica e esofagoscopia realizada nos cães anestesiados, foram fundamentais para confirmar a posição correta do cateter de pHmetria esofágica, e permitiram excluir o erro de posicionamento como uma variável que poderia influenciar os resultados. $\mathrm{O}$ posicionamento do cateter incorreto, tal como ocorreria com um cateter dobrado no lúmen esofágico ou com a sua ponta distante ou dentro do EGS ou do estômago, pode levar a falsas interpretações (5).

\section{CONCLUSÕES}

Este estudo permitiu concluir que:

1. A média da pHmetria esofágica intraluminal nos cães foi de $6.229+-0.5245$;

2. A incidência de refluxo gastroesofágico durante a anestesia com cetamina, midazolan e metadona, propofol e isoflurano foi de 11,11\%; e em um paciente submetido a ovariosalpingohisterectomia.

3. Não há alterações significativas do pH esofágico ao longo do tempo de recuperação anestésica com o protocolo utilizado.

Apoio e financiamento Agência: Conselho Nacional de Desenvolvimento Científico e Tecnológico 


\section{REFERÊNCIAS}

1. Pohl D, Tutuian R. Reflux monitoring: $\mathrm{pH}$-metry, Bilitec and oesophageal impedance measurements. Best Pract Res Clin Gastroenterol. 2009;23:299-311.

2. Nasi A, Moraes-Filho JPP, Cecconello I. Doença do refluxo gastroesofágico: revisão ampliada. Arq Gastroenterol. 2006;43:334-41.

3. Alarcón CAR, Ruiz DMB, Barreno RR, Díaz G, Casaús JMU, Herrera RG, et al. Gastroesophageal reflux in anesthetized dogs: a review. Rev Colomb Cienc Pecu. 2015;28:144-55.

4. Waterman AE, Hashim MA. Measurement of the length and position of the lower oesophageal sphincter by correlation of external measurements and radiographic estimations in dogs. Vet Rec. 1991;129:261-4.

5. Lemme EM. Métodos de avaliação e interpretação. In: Nasi A, Michelsohn NH. Avaliação funcional do esôfago. São Paulo: Roca; 2001. p.228-310.

6. Raptopoulos D, Galatos AD. Gastro-oesophageal reflux during anaesthesia induced with either thiopentone or propofol in the dog. Vet Anaesth Analg. 1997;2:20-2.

7. Wilson DV, Evans AT, Mauer WA. Pre-anesthetic meperidine: associated vomiting and gastroesophageal reflux during the subsequent anesthetic in dogs. Vet Anaesth Analg. 2007;34:15-22.

8. Anagnostou TL, Savvas I, Kazakos GM, Ververidis HN, Haritopoulou MR, Rallis TS, et al. Effect of endogenous progesterone and oestradiol-17beta on the incidence of gastrooesophageal reflux and on the barrier pressure during general anaesthesia in the female dog. Vet Anaesth Analg. 2009;36:308-18.

9. Panti A, Bennett RC, Corletto F, Brearley J, Jeffery N, Mellanby RJ. The effect of omeprazole on oesophageal $\mathrm{pH}$ in dogs during anaesthesia. J Small Anim Pract. 2009;50:540-4.

10. Nehra D, Howell P, Williams CP, Pye JK, Beynon J. Toxic bile acids in gastrooesophageal reflux disease: influence of gastric acidity. Gut. 1999;44:598-602.

11. Galatos AD, Savas I, Prassinos NN, Raptopoulos D. Gastro-oesophageal reflux during thiopentone or propofol anaesthesia in the cat. J Vet Med A Physiol Pathol Clin Med. 2001;48:287-94.

12. Favarato ES, Souza MV, Costa PRS, Pompermayer LG, Favarato LSC, Ribeiro Júnior JI. Ambulatory esophageal pHmetry in healthy dogs with and without the influence of general anesthesia. Vet Res Commun. 2011;35:271-82.

13. Sideri AI, Galatos AD, Kazakos GM, Gouletsou PG. Gastro-oesophageal reflux during anaesthesia in the kitten: comparison between use of a laryngeal mask airway or an endotracheal tube. Vet Anaesth Analg. 2009;36:547-54. 
14. Girão VCC, Pinheiro DCS, Morais SM, Gioso MA. Efeito protetor do extrato etanólico de Lippia sidoides (Alecrim pimenta) nas gengivites marginais de cães. Cienc Anim. 2001;11:13-6.

15. Wilson DV, Evans AT, Mauer WA. Influence of metoclopramide on gastroesophageal reflux in anesthetized dogs. Am J Vet Res. 2006;67:26-31.

16. McLauchlan G, Rawlings JM, Lucas ML, McCloy RF, Crean GP, McColl KE. Electrodes for 24 hours $\mathrm{pH}$ monitoring-a comparative study. Gut. 1987;28:935-9.

17. Wilson DV, Evans AT, Miller R. Effects of preanesthetic administration of morphine on gastroesophageal reflux and regurgitation during anesthesia in dogs. Am J Vet Res. 2005;66:386-90.

18. Wilson DV, Boruta DT, Evans AT. Influence of halothane, isoflurane and sevoflurane on gastroesophageal reflux during anesthesia in dogs. Am J Vet Res. 2006;67:1821-5.

19. Galatos AD, Raptopoulos D. Gastro-oesophageal reflux during anaesthesia in the dog: the effect of age, positioning and type of surgical procedure. Vet Rec. 1995;137:513-6.

20. Garcia CDM, Pinchbeck GL, Dugdale A, Senior JM. Retrospective study of the risk factors and prevalence of regurgitation in dogs undergoing general anaesthesia. Open Vet Sci J. 2013;7:6-11.

21. Rodríguez-Alarcón CA. Estudio retrospectivo y prospectivo de las endoscopias del tracto digestivo en pequeños animales [tese]. Cáceres: Facultad de Veterinaria, Universidad de Extremadura; 2010.

22. Hall AW, Moossa AR, Clark J, Cooley GR, Skinner DB. The effects of premedication drugs on the lower oesophageal high pressure zone and reflux status of rhesus monkeys and man. Gut. 1975;16:347-52.

23. Tanishima Y, Fujita T, Suzuki Y, Kawasaki N, Nakayoshi T, Tsuiboi K, et al. Effects of half-solid nutrients on gastroesophageal reflux in beagle dogs with or without cardioplasty and intrathoracic cardiopexy. J Surg Res. 2010;161:272-7.

24. Andenplas Y, Salvatore S, Hauser B. The diagnosis and management of gastrooesophageal reflux in infants. Early Hum Dev. 2005;81:1011-24.

25. Silva JMB, Silva PEMR, Brunelli MMC. pH metria esofágica abreviada. Correlación de valores del índice de reflujo (IR) en exámenes de 8 y 24 hs. Rev Cir Infant. 1996;6:20812.

26. Carneluti R, Martins JL, Cury EK. Esophageal pH monitoring study of eight hours in pediatric patients with suspected gastroesophageal reflux. Acta Cir Bras. 2005;20:116-20.

Recebido em: 14/08/2016 Aceito em: 03/08/2017 